Draft Version November 2, 2018

Preprint typeset using $\mathrm{LAT}_{\mathrm{E}} \mathrm{X}$ style emulateapj v. 11/12/01

\title{
X-RAY BURSTS FROM THE TERZAN 5 TRANSIENT IGR J17480-2446: NUCLEAR RATHER THAN GRAVITATIONAL
}

\author{
Manoneeta Chakraborty $^{1}$ and Sudip Bhattacharyya ${ }^{1}$ \\ Draft version November 2, 2018
}

\begin{abstract}
The 2010 outburst of the transient neutron star low-mass X-ray binary IGR J17480-2446 has exhibited a series of unique X-ray bursts, as well as millihertz $(\mathrm{mHz})$ quasi-periodic oscillations (QPOs) related to these bursts. It has been recently proposed that these are type-II bursts, powered by the gravitational energy. This implies that the current nuclear-burning based model of $\mathrm{mHz}$ QPOs is not correct, and this timing feature cannot be used as a tool to measure the neutron star parameters. We report the analysis of the Rossi X-ray Timing Explorer data of IGR J17480-2446 to show that the burst properties of this source are quite different from the properties of the type-II bursts observed from the rapid burster and GRO J1744-28. For example, the inferred ratio $(\sim 50-90)$ of the non-burst fluence to burst fluence is consistent with the thermonuclear origin of IGR J17480-2446 bursts, and is significantly different from this ratio $(\lesssim 4)$ for type-II bursts. Our results suggest that the bursts and the $\mathrm{mHz}$ QPOs from IGR J17480-2446 are powered by the nuclear energy.
\end{abstract}

Subject headings: accretion, accretion disks — stars: neutron - X-rays: binaries - X-rays: bursts X-rays: individual: IGR J17480-2446 - X-rays: stars

\section{INTRODUCTION}

The first observed outburst (Bordas et al. 2010) of the globular cluster Terzan 5 source IGR J17480-2446 in Oct/Nov, 2010 revealed that it is a neutron star low-mass X-ray binary (LMXB) and an $11 \mathrm{~Hz}$ pulsar Chenevez et al. 2010; Strohmaver and Markwardt 2010). An X-ray burst was observed from this source with Rossi X-ray Timing Explorer (RXTE) on Oct 13, 2010 (Strohmaver and Markwardt 2010). While this is not surprising even for a pulsar, what made this source very interesting is the recurrent bursts observed for next few days (Altamirano et al. 2010b; Papitto et al. 2011). As the source intensity increased, the bursts became more frequent, and eventually disappeared, and millihertz $(\mathrm{mHz})$ quasi-periodic oscillations (QPOs) appeared (Altamirano et al. 2010b; Linares et al. 2010). Finally, the bursts reappeared, and as the source intensity decreased, they became less frequent (Chakraborty and Bhattacharyva 2010). The burst recurrence frequencies observed between October 13 and 16 converge asymptotically towards the $\mathrm{mHz} \mathrm{QPO}$ frequency, which suggests that these QPO is related to bursts (Linares et al. 2010). If these bursts are type-I or thermonuclear (Strohmaver and Bildsten 2006), then such a relation is expected from a widely accepted $\mathrm{mHz}$ QPO model, which interprets this timing feature as a signature of marginally stable nuclear burning (Heger et al. 2007). However, Galloway and in't Zand (2010) reported that, except the Oct 13 burst, no other burst up to Oct 26 showed a clear cooling trend during burst decay. This motivated them to suggest that, except the first burst, others are type-II bursts. The type-II bursts are believed to be caused by the accretion instability, and hence powered by the gravitational potential energy. This would mean that the $\mathrm{mHz}$ QPOs are related to the accretion dynamics, rather than nuclear burning, and hence plausibly could not be used as a tool to measure the neutron star parameters
(Heger et al. 2007; Bhattacharvva 2010). Type-II bursts have so far been observed from two sources: rapid burster and GRO J1744-28 (Tan et al. 1991; Lewin et al. 1995, 1996). Galloway and in't Zand (2010) have also indicated that IGR J17480-2446 might be a GRO J1744-28 analogue (since both have relatively wide orbit and slow pulsations), further strengthening the gravitational origin argument for the IGR J17480-2446 bursts. It is therefore extremely important to investigate whether the X-ray bursts from IGR J17480-2446 are powered by the nuclear energy or by the gravitational energy (1) in order to understand the nature of the unique source IGR J17480-2446, and its unique bursts, and (2) to find out the origin of $\mathrm{mHz}$ QPOs and their usefulness in understanding thermonuclear bursts and in measuring neutron star parameters.

We have performed spectral and timing analysis of the entire RXTE Proportional Counter Array (PCA) data from IGR J17480-2446, the details of which will be reported elsewhere. In this Letter, we report and discuss the results required to address the single very important question mentioned above.

\section{OBSERVATION AND DATA ANALYSIS}

The neutron star LMXB IGR J17480-2446 was observed almost everyday with $R X T E$ between Oct 13, 2010 and Nov 19, 2010, i.e., during its outburst. The total observation time was $\approx 297 \mathrm{ks}$ (proposal no. 95437; 46 obsIds: $95437-01-01-00$ to 95437-01-14-00). We have found $\sim 400$ bursts (including the very weak ones) in the RXTE data of this source, and analyzed the entire PCA data with the aim of understanding the nature of these unique bursts. In order to examine the lightcurves, and to measure the source properties such as burst duration, burst interval (time gap between two successive bursts) and spectral evolution during a burst, we have used the event-mode/good-Xenon files. This is because, this mode has sufficiently good time resolution and spectral resolution. In order to perform the time-resolved

1 Tata Institute of Fundamental Research, Mumbai-400005, India; manoneeta@tifr.res.in; sudip@tifr.res.in 
spectroscopy of the bursts, which could be comfortably distinguished from the non-burst emissions, we have divided each burst into segments with sufficient statistics. For each segment, we have created a total energy spectrum with deadtime correction (van der Klis 1989), and a background spectrum from the pre-burst emission Bhattacharyva and Strohmaver 2006; Galloway et al. 2008). Usually a blackbody model is used to describe a burst spectrum, and hence we have fitted each spectrum in $3-15 \mathrm{keV}$ with an absorbed blackbody (phabs*bbodyrad in XSPEC) for a fixed neutral hydrogen column density $N_{\mathrm{H}}=3.8 \times 10^{22} \mathrm{~cm}^{-2}$ (Galloway and in't Zand 2010; Kuulkers et al. 2003). The blackbody provides acceptable fits (for the degrees of freedom $\nu=17$ ) with $\chi^{2} / \nu \leq$ 1.0 for $\sim 40 \%$ segments and $1<\chi^{2} / \nu \leq 1.5$ for $\sim 45 \%$ segments, excluding a few segments with $\chi^{2} / \nu>2.0$. We have used these fits to infer the total energy (timeintegrated flux or fluence), as well as the evolution of temperature, emitting area and flux of each burst. We have also fitted the deadtime corrected non-burst spectra in $3-15 \mathrm{keV}$, using backgrounds created from the RXTE data analysis tool PCABACKEST. These spectra were made from standard-2 mode files. First we have tried the conventionally used thermal models absorbed blackbody (phabs*bbodyrad in XSPEC) and absorbed diskblackbody (phabs*diskbb in XSPEC), as well as the nonthermal model absorbed powerlaw (phabs*powerlaw in XSPEC), in order to fit the non-burst spectra. Similar to the burst spectral fitting, we have fixed $N_{\mathrm{H}}$ at $3.8 \times 10^{22}$ $\mathrm{cm}^{-2}$. Each of these single component models gave extremely bad fit. Next we have tried three two-component models combining two of bbodyrad, diskbb and powerlaw in rotation. The phabs*(bbodyrad+powerlaw) model gave much better fit than phabs*(bbodyrad+diskbb), and somewhat better fit than phabs* (diskbb+powerlaw). Furthermore, the addition of a Gaussian component describing an iron $\mathrm{K} \alpha$ emission line between $6.4-6.97$ $\mathrm{keV}$ improves the fit significantly (e.g., significance $\approx$ $1-5.6 \times 10^{-4}$ for the Oct 26, 2010 data), and gives acceptable fits (see also Chakraborty et al. (2011)). Therefore, we have fitted each non-burst spectrum with the phabs*(bbodyrad+powerlaw+Gaussian) model in order to infer the flux, fluence and other spectral parameters. We have tried to estimate the contributions of the field sources to this non-burst flux. The total 2-6 keV Chandra count rate of all the sources (except CX25, which is IGR J17480-2446; Pooley et al. (2010)) given in Tables 1 and 2 of Heinke et al. (2006) is $\approx 0.06 \mathrm{cps}$, which is about $0.1 \%$ of the $2-6 \mathrm{keV}$ Chandra count rate equivalent to the lowest (of Oct 13, 2010) observed RXTE PCA flux. This shows that the non-burst flux has been overestimated by $\lesssim 0.1 \%$.

\section{RESULTS AND DISCUSSION}

In this Letter, we report the evolution of various burst and non-burst properties of IGR J17480-2446 throughout the source outburst. Examples of a few days given in Table 1 and Fig. 1 show that the burst properties are well correlated with the non-burst intensity. The Oct 13 burst detected with $R X T E$ was likely to be powered by nuclear energy because of the following reasons. (a) The burst light curve rises rapidly and decays slowly like a typical ther- monuclear burst (Fig. 11). (b) The blackbody temperature decreases during burst decay Gallowav and in't Zand 2010). (c) Intensity variation of this burst at the pulsar frequency was detected (Altamirano et al. 2010a), and this feature is broader in power spectrum during the burst than in the non-burst portions. Here we discuss the properties of the bursts following the Oct 13 burst, in order to find out whether these bursts are powered by nuclear energy or gravitational energy.

(1) Burst spectrum vs. non-burst spectrum: The burst spectrum of IGR J17480-2446 is typically well described with a blackbody $(\S 2)$, and the addition of a powerlaw does not improve the fit (note that, for fainter bursts, this might be due to insufficient statistical quality). The non-burst spectrum of IGR J17480-2446 cannot be fitted with a blackbody, and a blackbody plus powerlaw model is required (see $\S 2$, see also Chakraborty et al. (2011)). So these two spectra are plausibly sufficiently different, indicating different origins. However, these two spectra are similar to each other for GRO J1744-28 (Lewin et al. 1996).

(2) Temperature evolution during burst decay: Most of the bursts from IGR J17480-2446 do not exhibit a clear cooling trend during the burst decay. However, this cannot rule out the nuclear origin of the bursts for the following reasons. (a) The Nov 18 burst shows a cooling trend with a significance of $\approx 1-9 \times 10^{-8}$, while the similar bursts of Nov 19 do not show such a trend. (b) Several likely thermonuclear bursts (e.g., 18th burst of EXO 0748-676 in Galloway et al. (2008)) did not show a cooling trend. (c) If the color factor $f$ increases with the decrease of the effective temperature $T_{\text {eff }}$ (may be possible for high metalicity of burning matter, see Table 2 of Majczyna et al. $(2005))$, the cooling trend may not be visible, as the color temperature $T_{\mathrm{c}}=f T_{\text {eff }}$.

(3) Ratio of non-burst fluence to burst fluence: For a thermonuclear burst, the ratio of the nuclear energy generated to the gravitational energy released is expected to be $\sim 40$ (Strohmaver and Bildsten 2006). The ratio of non-burst fluence to burst fluence gives an upper limit of this energy ratio, as stable nuclear burning can happen during the non-burst time. The ratio of the fluences ( 50 - 90) inferred for IGR J17480-2446 bursts throughout the outburst is very consistent with the plausible thermonuclear origin of these bursts (see Table 11). However, this ratio is significantly smaller than 40 for rapid burster $(\lesssim 1$; Tan et al. (1991)) and GRO J1744-28 ( $\lesssim 4$; Lewin et al. (1996)), indicating that the type-II bursts from these sources are not of thermonuclear origin. Note that, although we have estimated the ratio of the fluences for IGR J17480-2446 in the $3-15 \mathrm{keV}$ range, the bolometric ratio would also be consistent with the expected value $\sim 40$ because of the following reasons. (a) Since the non-burst spectrum has two continuum components (a blackbody similar to the burst spectrum, plus a powerlaw), a larger portion of the non-burst energy is likely to be outside the $3-15 \mathrm{keV}$ range compared to the burst energy. (b) Even if the entire non-burst energy is in the $3-15 \mathrm{keV}$ range (which is the conservative scenario), our estimated ratio will be less by a small factor of $\approx 1.2$ for a typical burst blackbody temperature of $2 \mathrm{keV}$.

(4) Burst shape and duration: the bursts neither show 
"ringing" nor "flat top" like the type-II bursts of the rapid burster (Tan et al. 1991). Their duration gradually changes (Table 1) unlike the GRO J1744-28 bursts (Fishman et al. 1996). Fig. 22 clearly shows that the shapes of the bursts are similar to the that of the Oct 13 burst, and any change is gradual throughout the outburst. This would not happen if the Oct 13 burst and the other bursts originated from two independent physical mechanisms. These points support the nuclear origin of the IGR J17480-2446 bursts.

(5) Intensity dips after bursts: while type-II bursts from the rapid burster and GRO J1744-28 are followed by intensity dips (Tan et al. 1991; Lewin et al. 1996), no clear evidence of such dips has been found for IGR J17480-2446 bursts. Only $10 \%$ bursts are followed by weak dips, which are plausibly of the non-burst origin.

(6) Burst fluence versus burst interval: burst fluence is positively correlated with burst interval for IGR J174802446 (Table 1), while the fluence of GRO J1744-28 type-II bursts remained approximately constant when the burst interval changed by factors of $\sim 4$ (Kouveliotou et al. 1996).

(7) Burst peak flux and burst fluence versus non-burst flux: while with the increase of non-burst flux, both burst peak flux and burst fluence of GRO J1744-28 increase (Lewin et al. 1996), these parameter values of IGR J17480-2446 decrease (Table 1).

\section{CONCLUSION}

The gradual (as opposed to abrupt) change of all the burst properties throughout the outburst (Table 1 and Fig. 11) strongly indicate that all the bursts from IGR J17480-2446, including the Oct 13 burst, originate from the same physical mechanism. The points 3,4 and 5 of $\S 3$ suggest that these bursts are of thermonuclear origin, and $\mathrm{mHz}$ QPOs, plausibly caused by the quasi-stable nuclear burning, can be used as a tool to measure the neutron star parameters. The point 2 of $\S 3$ indicates that a clear cooling may not always be present during a thermonuclear burst decay. The points $1,3,4,5,6$ and 7 of $\S 3$ show that IGR J17480-2446 is not a GRO J1744-28 analogue ( $($ 1). Finally, we note that the IGR J17480-2446 bursts are similar to the GS 1826-238 bursts in their clock-like recurrence times and in the shortening of the recurrence time with the non-burst flux increase (Strohmaver and Bildsten 2006). ${ }^{1}$

We thank Arunava Mukherjee, Jon Miller and Tod Strohmayer for discussion, and an anonymous referee for constructive comments. This work was supported in part by the TIFR plan project 11P-408 (P.I.:K. P. Singh).

${ }^{1}$ After this Letter was submitted and made public, the following papers on the bursts from IGR J17480-2446 were submitted in the arXiv: Cavecchi et al. (2011), Linares et al. (2011). These papers supported our conclusion regarding the thermonuclear origin of these bursts.

\section{REFERENCES}

Altamirano, D., Homan, J., Linares, M., et al. 2010b, ATel, 2952, 1

Altamirano, D., Watts, A., Kalamkar, M., et al. 2010a, ATel, 2932, 1

Bhattacharyya, S. 2010, Advances in Space Research, 45, 949

Bhattacharyya, S., \& Strohmayer, T. E. 2006, ApJ, 641, L53

Bordas, P., Kuulkers, E., Alfonso-Garzón, J., et al. 2010, ATel, 2919, 1

Cavecchi, Y., Patruno, A., Haskell, B., Watts, A. L., Levin, Y., Linares, M., Altamirano, D., Wijnands, R., \& van der Klis, M., 2011, arXiv: 1102.1548

Chakraborty, M., \& Bhattacharyya, S. 2010, ATel, 3044, 1

Chakraborty, M., Bhattacharyya, S., \& Mukherjee, A. 2011, arXiv: 1102.1033 v 1

Chenevez, J., Kuulkers, E., Alfonso-Garzón, J., et al. 2010, ATel, 2924, 1

Fishman, G. J., et al. 1996, IAU Circ., No. 6290

Galloway, D. K., \& in't Zand, J. J. M. 2010, ATel, 3000, 1

Galloway, D. K., Muno, M. P., Hartman, J. M., Psaltis, D., \& Chakrabarty, D. 2008, ApJS, 179, 360

Heger, A., Cumming, A., \& Woosley, S. E. 2007, ApJ, 665, 1311

Heinke, C. O., Wijnands, R., Cohn, H. N., Lugger, P. M., Grindlay, J. E., Pooley, D., \& Lewin, W. H. G. 2006, ApJ, 651, 1098

Kouveliotou, C., van Paradijs, J., Fishman, G. J., et al. 1996, Nature, 379,399
Kuulkers, E., den Hartog, P. R., in't Zand, J. J. M., Verbunt, F. W. M., Harris, W. E., \& Cocchi, M. 2003, A\&A, 399, 663

Lewin, W. H. G., Rutledge, R. E., Kommers, J. M., van Paradijs, J., \& Kouveliotou, C. 1996, ApJ, 462, L39

Lewin, W. H. G., van Paradijs, J., \& Taam, R. E. 1995, in X-Ray Binaries, ed. W. H. G. Lewin, J. van Paradijs, \& E. P. J. van den Heuvel (Cambridge: Cambridge Univ. Press), 175

Linares, M., Altamirano, D., Watts, A., et al. 2010, ATel, 2958, 1

Linares, M., Chakrabarty, D., van der Klis, M., arXiv:1102.1455

Majczyna, A., Madej, J., Joss, P. C., \& Róaska, A., 2005, A\&A, 430, 643

Papitto, A., D'Aí, A., Motta, S., et al. 2011, A\&A, 526, L3

Pooley, D., Homan, J., Heinke, C., Linares, M., Altamirano, D., \& Lewin W. 2010, ATel, 2974, 1

Strohmayer, T.E., \& Bildsten, L. 2006, in Compact Stellar X-ray Sources, ed. W.H.G. Lewin and M. van der Klis, (Cambridge University Press: Cambridge), 39, 113

Strohmayer, T. E., \& Markwardt, C. B. 2010, ATel, 2929, 1

Tan, J., Lewin, W. H. G., Lubin, L. M., et al. 1991, MNRAS., 251, 1

van der Klis, M. 1989, in Timing Neutron Stars, ed. H. Ögelman and E. P. J. van den Heuvel, (Kluwer Academic / Plenum Publishers: New York), 27 
TABLE 1

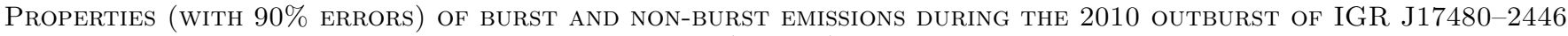
(SEE $\S 3$ ).

\begin{tabular}{ccccccccc}
\hline Date $^{1}$ & $\begin{array}{c}\text { Time } \\
\text { range }^{2}\end{array}$ & $\begin{array}{c}\text { Non-burst } \\
\text { flux }\end{array}$ & $\begin{array}{c}\text { Burst } \\
\text { peak flux }\end{array}$ & $\tau^{5}$ & T $_{\text {int }}{ }^{6}$ & $\begin{array}{c}\text { Burst } \\
\text { fluence }\end{array}$ & $\begin{array}{c}\text { Ratio of } \\
\text { fluences }^{8}\end{array}$ & $\begin{array}{c}\text { No. of } \\
\text { bursts }^{9}\end{array}$ \\
\hline Oct 13 & $00: 12: 32-01: 05: 10$ & $2.58_{-0.02}^{+0.01}$ & $9.89_{-0.78}^{+0.73}$ & 120 & - & $26.70_{-0.54}^{+0.52}$ & - & 1 \\
Oct 14 & $04: 28: 01-05: 24: 09$ & $8.62_{-0.04}^{+0.04}$ & $5.36_{-0.56}^{+0.48}$ & 105 & 1034 & $15.35_{-0.67}^{+0.61}$ & $58.11_{-2.55}^{+2.32}$ & 3 \\
Oct 15 & $10: 24: 32-11: 06: 11$ & $10.65_{-0.04}^{+0.04}$ & $2.73_{-0.23}^{+0.02}$ & 72 & 512 & $6.60_{-0.29}^{+0.22}$ & $82.62_{-3.59}^{+2.76}$ & 5 \\
Oct 23 & $03: 26: 40-04: 14: 09$ & $11.81_{-0.06}^{+0.05}$ & $2.70_{-0.39}^{+0.29}$ & 56 & 434 & $6.72_{-0.44}^{+0.37}$ & $76.38_{-4.99}^{+4.25}$ & 6 \\
Oct 26 & $08: 19: 28-09: 15: 11$ & $10.55_{-0.05}^{+0.04}$ & $4.21_{-0.31}^{+0.27}$ & 60 & 722 & $11.21_{-0.35}^{+0.31}$ & $67.94_{-2.15}^{+1.92}$ & 5 \\
Oct 31 & $09: 05: 20-10: 02: 10$ & $9.54_{-0.04}^{+0.04}$ & $4.18_{-0.42}^{+0.38}$ & 90 & 1016 & $10.31_{-0.42}^{+0.37}$ & $93.96_{-3.81}^{+3.43}$ & 3 \\
Nov 05 & $06: 43: 28-07: 40: 10$ & $8.92_{-0.04}^{+0.04}$ & $4.56_{-0.46}^{+0.41}$ & 100 & 1273 & $12.50_{-0.38}^{+0.35}$ & $90.83_{-2.82}^{+2.58}$ & 3 \\
Nov 08 & $05: 17: 20-06: 14: 10$ & $8.35_{-0.04}^{+0.04}$ & $4.92_{-0.60}^{+0.54}$ & 120 & 1488 & $17.62_{-0.46}^{+0.42}$ & $70.48_{-1.85}^{+1.72}$ & 2 \\
Nov 15 & $03: 32: 32-04: 29: 09$ & $7.26_{-0.03}^{+0.03}$ & $7.65_{-0.63}^{+0.59}$ & 130 & 2137 & $26.85_{-0.59}^{+0.55}$ & $57.80_{-1.30}^{+1.20}$ & 2 \\
Nov 18 & $02: 06: 24-03: 03: 11$ & $6.62_{-0.02}^{+0.02}$ & $9.17_{-0.65}^{+0.61}$ & 135 & 2391 & $36.41_{-0.70}^{+0.65}$ & $43.49_{-0.85}^{+0.79}$ & 2 \\
\hline
\end{tabular}

${ }^{1}$ Date of observation.

${ }^{2}$ Start and end times (UTC) of observation.

${ }^{3}$ Average flux (in $10^{-9}$ ergs $\mathrm{cm}^{-2} \mathrm{~s}^{-1}$ ) within $3-15 \mathrm{keV}$ in the portions of the data without bursts.

${ }^{4}$ Average flux (in $10^{-9}$ ergs $\mathrm{cm}^{-2} \mathrm{~s}^{-1}$ ) within $3-15 \mathrm{keV}$ of the peak of a burst.

${ }^{5}$ Average duration (in s) of a burst (from the time corresponding to $\sim 5 \%$ of peak count rate during rise to the time corresponding to $\sim 5 \%$ of peak count rate during decay).

${ }^{6}$ Average time gap (burst interval; in s) between two successive bursts.

${ }^{7}$ Average total energy (in $10^{-8}$ ergs $\mathrm{cm}^{-2}$ ) within $3-15 \mathrm{keV}$ of a burst.

${ }^{8}$ Ratio of the non-burst fluence to the corresponding burst fluence.

${ }^{9}$ Number of bursts within the given time range. 


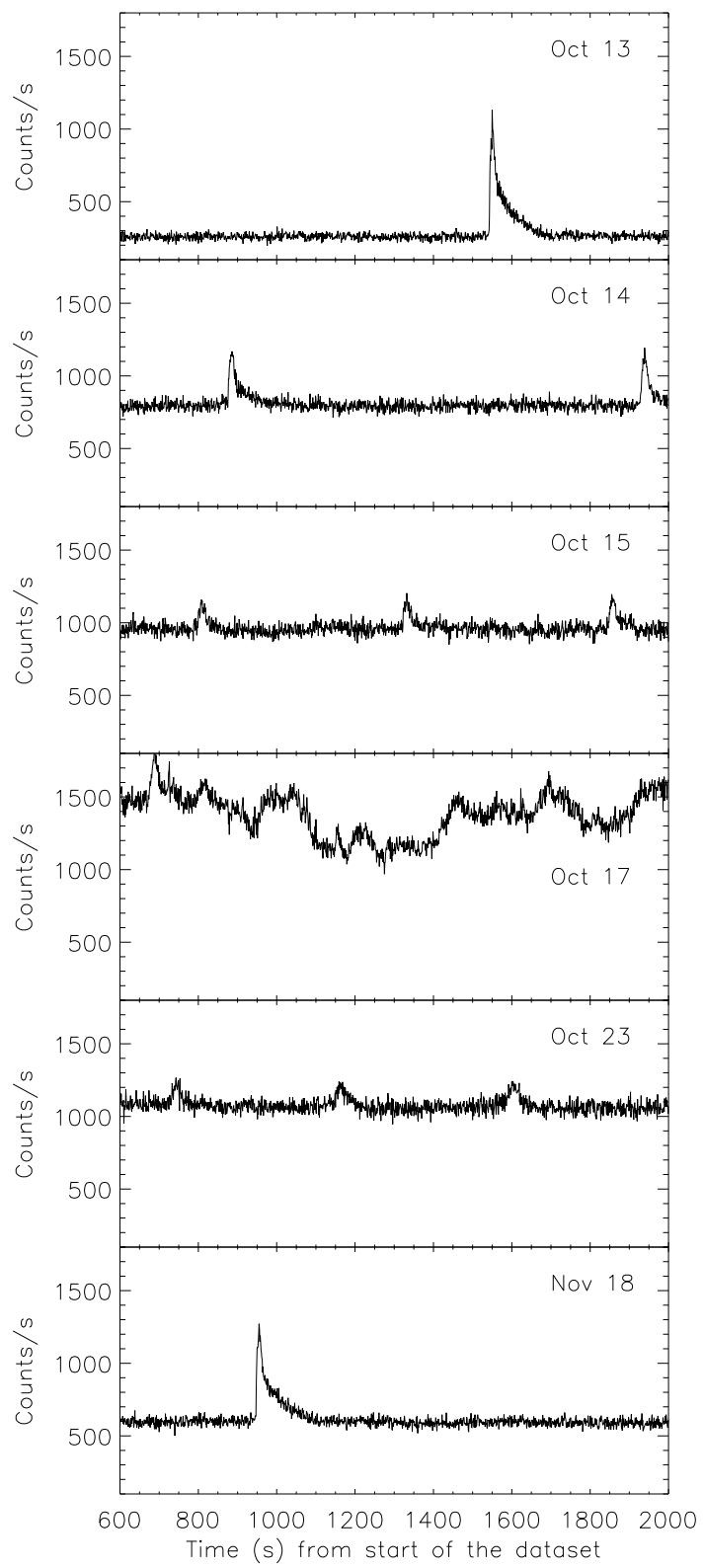

FIG. 1.- Light curves of $1 \mathrm{~s}$ resolution of portions of six $R X T E$ PCU-2 continuous data sets from IGR J17480-2446. The date of observation of each data set is mentioned. This figure shows that during the rise of the 2010 outburst, as the non-burst flux increases, the burst peak flux and the burst interval gradually decrease. The initial burst properties return as the source intensity decays $(\S[$ ). 


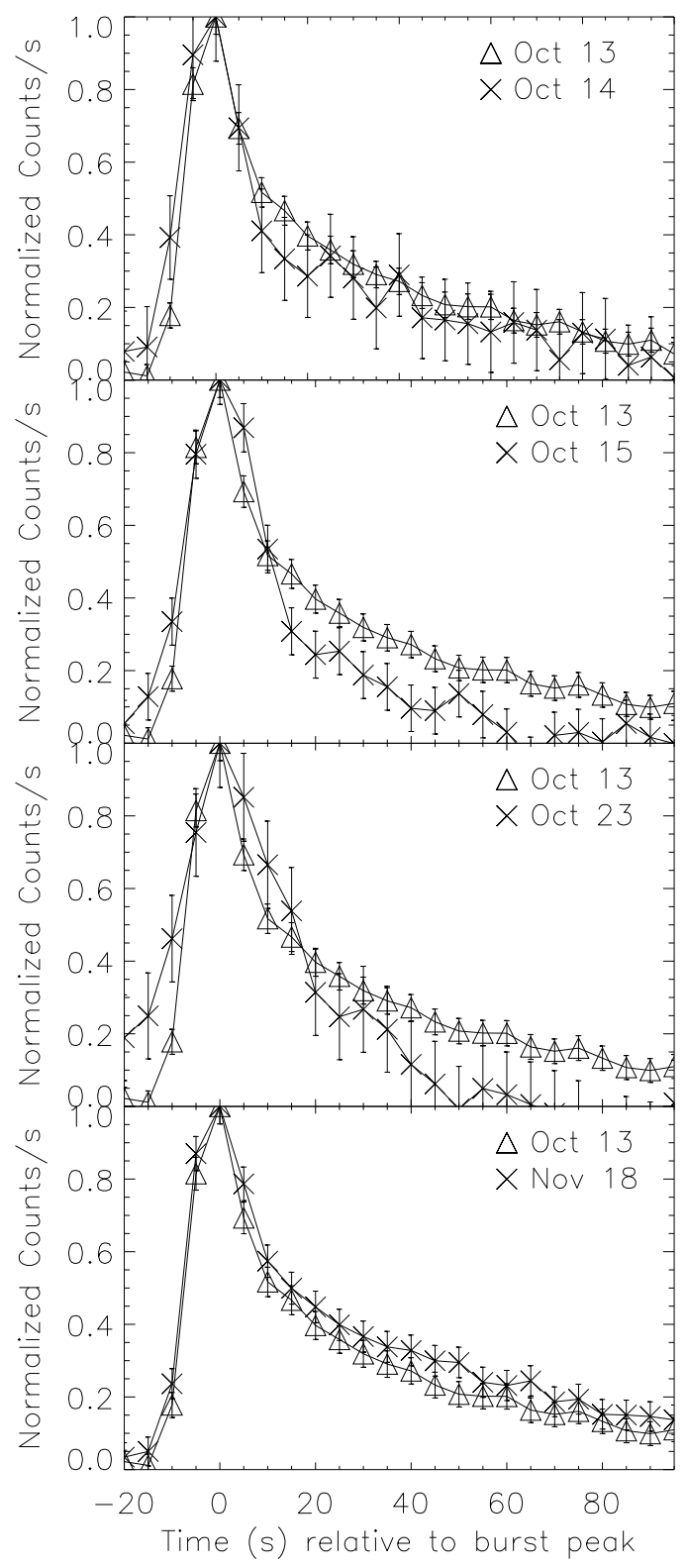

FIG. 2.- Comparisons of the bursts of Oct 14, Oct 15, Oct 23 and Nov 18 with the Oct 13 burst. Each burst light curve is pre-burst level subtracted and normalized with the peak count rate. In case of Oct 15, four burst light curves are combined to make the statistics better. In case of Oct 23 , six burst light curves are combined. This figure shows that the shapes of the bursts of different days are not drastically different from each other, and the gradual change of the shape is correlated with various burst and non-burst properties (compare with Table 1 and Fig. 1). 